\title{
Apoptosis of stem cells likely determines the manifestation of type 2 diabetic nephropathy: predictive and preventive potentials
}

\author{
Mahmood S. Mozaffari", Jun Yao Liu, Jack C. Yu, Babak Baban \\ From EPMA-World Congress 2013 \\ Brussels, Belgium. 20-21 September 2013
}

\section{Scientific objective}

One of the most pressing health-related challenges is the worldwide epidemic of type 2 diabetes and associated complications such as nephropathy. Indeed, type 2 diabetic patients account for the majority of individuals with chronic kidney disease requiring renal replacement therapy. This not only suggests inadequacy of current drug therapies but also failure of endogenous protective mechanisms. Stem cells are known to play pivotal roles in repair and regeneration of damaged/dysfunctional tissues. Our initial observations suggested that initiation and progression of type 2 diabetes mellitus is associated with progressive reduction in endothelial progenitor cells in the obese diabetic $\mathrm{db} / \mathrm{db}$ mice. Thus, the present study was intended to more fully explore the status of components of stem cells in the setting of type 2 diabetic nephropathy and whether the diabetic milieu affects the survivability of these cells.

\section{Technical approach/methods}

Male 16-week-old obese diabetic $\mathrm{db} / \mathrm{db}$ mice (and their $\mathrm{db} / \mathrm{m}$ controls) were used for this study. Accordingly, indices of glycemic status (e.g., insulin resistance index, hemoglobin A1c), kidney function (e.g., albuminuria, creatinine clearance) were determined in the context of assessment of components of stem cells in peripheral blood and renal tissue. In addition, we measured apoptotic cell death in components of stem cells.

\section{Results/interpretation}

The $\mathrm{db} / \mathrm{db}$ mice showed significant increases in insulin resistance index and hemoglobin A1c in association with marked increase in albuminuria but reduction in

\footnotetext{
* Correspondence: MMOZAFFA@gru.edu

Department of Oral Biology and Plastic Surgery, Georgia Regents University, Augusta, Georgia 30912, USA
} 